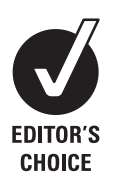

See Editorial, pg 1521

${ }^{1}$ Arthritis Research UK Pain Centre, Academic Rheumatology, Nottingham City Hospital, UK

${ }^{2}$ Nottingham Digestive Diseases Centre and Biomedical Research Unit, Queens Medical Centre, Nottingham, UK

${ }^{3}$ Worldwide Clinical Trials, Nottingham, UK

${ }^{4}$ Creativeclinical, Nottingham, UK

${ }^{5}$ Hill PharmaConsulting Ltd, Uttoxeter, UK

${ }^{6}$ Reckitt Benckiser Healthcare UK Ltd, Hull, UK

${ }^{7}$ Clearcut Clinical Consulting,

Nottingham, UK

\section{Correspondence to}

Michael Doherty, Arthritis Research UK Pain Centre, Academic Rheumatology, Clinical Sciences Building,

City Hospital,

Nottingham NG5 1PB, UK: michael.doherty@nottingham. ac.uk

Accepted 12 July 2011

\title{
A randomised controlled trial of ibuprofen, paracetamol or a combination tablet of ibuprofen/ paracetamol in community-derived people with knee pain
}

\author{
Michael Doherty, ${ }^{1}$ Chris Hawkey, ${ }^{2}$ Michael Goulder, ${ }^{3}$ lain Gibb, ${ }^{4}$ Nicola Hill, ${ }^{5}$ \\ Sue Aspley, ${ }^{6}$ Sandie Reader ${ }^{7}$
}

\begin{abstract}
Objectives To compare the efficacy and safety of single versus combination non-prescription oral analgesics in community-derived people aged 40 years and older with chronic knee pain.
\end{abstract}

Methods A randomised, double-blind, four-arm, parallelgroup, active controlled trial investigating short-term (day 10) and long-term (week 13) benefits and sideeffects of four regimens, each taken three times a day: ibuprofen $(400 \mathrm{mg})$; paracetamol $(1000 \mathrm{mg})$; one fixeddose combination tablet (ibuprofen $200 \mathrm{mg} /$ paracetamol $500 \mathrm{mg}$ ); two fixed-dose combination tablets (ibuprofen 400 mg/paracetamol $1000 \mathrm{mg}$ ).

Results There were 892 participants (mean age 60.6, range 40-84 years); 63\% had radiographic knee osteoarthritis and $85 \%$ fulfilled American College of Rheumatology criteria for osteoarthritis. At day 10, two combination tablets were superior to paracetamol $(p<0.01)$ for pain relief (determined by mean change from baseline in WOMAC pain; $n=786$ ). At 13 weeks, significantly more participants taking one or two combination tablets rated their treatment as excellent/ good compared with paracetamol $(p=0.015, p=0.0002$, respectively; $n=615$ ). The frequency of adverse events was comparable between groups. However, by 13 weeks, decreases in haemoglobin ( $\geq 1 \mathrm{~g} / \mathrm{dl})$ were observed in some participants in all groups. Twice as many participants taking two combination tablets had this decrease compared with those on monotherapy ( $p<0.001$; paracetamol, 20.3\%; ibuprofen, $19.6 \%$; one or two combination tablets, $24.1 \%, 38.4 \%$, respectively).

Conclusions Ibuprofen/paracetamol combination analgesia, at non-prescription doses, confers modest short-term benefits for knee pain/osteoarthritis. However, in this population, paracetamol $3 \mathrm{~g} /$ day may cause similar degrees of blood loss as ibuprofen $1200 \mathrm{mg} / \mathrm{day}$, and the combination of the two appears to be additive.

Study no ISRCTN77199439

Knee pain affects $25-37 \%$ of people aged over 50 years, ${ }^{1-6}$ approximately $50 \%$ of whom have, or will develop, radiographic osteoarthritis. 78 Many people $(28-33 \%)$ self-manage their pain with non-prescription analgesics, ${ }^{1} 69$ most commonly paracetamol and non-steroidal anti-inflammatory drugs (NSAIDs). Current osteoarthritis treatment guidelines recommend paracetamol as a first choice oral analgesic, largely because it lacks the gastrointestinal side-effects associated with NSAID. ${ }^{10-13}$ Nevertheless, systematic reviews confirm NSAIDs are superior to paracetamol at improving pain and functional status in osteoarthritis. ${ }^{1415}$ The addition of a second analgesic is recommended when pain is insufficiently controlled by paracetamol, ${ }^{10-13}$ but there are few studies, and few data, on combination analgesic therapy for knee pain/osteoarthritis. ${ }^{11} 12$

The combination of analgesics with different modes of action has the potential to offer enhanced pain relief with reduced dosage and consequently improved tolerability. ${ }^{16}$ Ibuprofen and paracetamol are considered to differ in their modes of action and are therefore candidates to be used in combination analgesia. Ibuprofen is a NSAID that inhibits cyclooxygenase enzymes. ${ }^{17}$ The mode of action of paracetamol in not completely understood; it is thought to inhibit a subclass of cyclooxygenase enzyme isoforms in the central nervous system, which may involve cyclooxygenase 3 , and some cyclooxygenase 2, inhibition. ${ }^{18-20}$ Both ibuprofen and paracetamol have been shown to confer additional efficacy when used in combination with codeine, in the short to medium term. ${ }^{21-25}$ However, the side-effects and tolerability issues associated with codeine make it unsuitable for many patients. ${ }^{26}$

The objective of this study was to determine the efficacy and tolerability of a novel fixed-dose combination tablet of ibuprofen/paracetamol in community-derived participants with chronic knee pain. Studies on regional musculoskeletal pain and osteoarthritis often focus on hospital-referred patients, with a bias towards more severe disease in randomised controlled clinical trials. Consequently, patients enrolled in those studies are not representative of those who take non-prescription analgesics and the data cannot confidently be generalised to this wider patient population. As this novel combination tablet is intended for non-prescription use, a community population was chosen for this study, to reflect accurately people who self-medicate their pain. Short-term efficacy and safety were measured at day 10 - the usual recommended period for selfmedication with non-prescription drugs. However, the trial was continued for 13 weeks, to investigate efficacy and safety in people who self-medicate for longer than recommended.

\section{METHODS}

\section{Design and subject selection}

This was a randomised, double-blind, four-arm, parallel-group, active controlled study lasting 
3 months. Participants had knee pain but were not under medical supervision for the condition. It was conducted in accordance with the Declaration of Helsinki (version 4; 1996), International Conference on Harmonization-good clinical practice standards and was approved by the appropriate research ethics committees (trial no: ISRCTN77199439). All participants provided written, informed consent.

Most participants were recruited from Nottinghamshire by postal questionnaire or local press/radio advertisements and the remainder by a site management organisation (Synexus Clinical Research, UK), by press/radio advertisements and general practitioner identification. Inclusion criteria were: age 40 years and older; knee pain for most of the past 3 months and on 4 of 7 preceding days; discontinuation of current analgesics; Steinbrocker functional capacity classification of I-III; ${ }^{27}$ pain affecting the index knee (after a washout period if currently taking analgesics) of $30 \mathrm{~mm}$ or greater and $80 \mathrm{~mm}$ or less on a $100-\mathrm{mm}$ visual analogue scale over the previous $48 \mathrm{~h}$ for one or more of the following: walking on a flat surface, going up/down stairs, at night, sitting, lying, standing upright. Exclusion criteria were: concomitant rheumatic, malignant, gastrointestinal, renal or hepatic conditions; taking anticoagulants (except $\leq 325 \mathrm{mg}$ aspirin/day); recent receipt of any drug to modify joint structure/function.

\section{Randomisation and masking}

Eligible participants were randomly assigned using a computer-based schedule; stratified according to the presence/absence of radiographic osteoarthritis. Drugs were over-encapsulated in size AA-EL capsules (Capsugel, Bornem, Belgium). Treatment groups were: two ibuprofen $200 \mathrm{mg}$ capsules; two paracetamol $500 \mathrm{mg}$ capsules; one ibuprofen $200 \mathrm{mg} /$ paracetamol $500 \mathrm{mg}$ capsule plus one placebo capsule; two ibuprofen $200 \mathrm{mg} /$ paracetamol $500 \mathrm{mg}$ capsules. Participants were instructed to take medication three times a day with water ( $\geq 6 \mathrm{~h}$ between doses).

\section{Assessments}

Baseline assessments included: demographic data; medical/ medication history; general health assessment including haematology and biochemistry analyses; examination for the presence and size of knee effusion, and features of osteoarthritis (restriction, crepitus, bony swelling); posterior-anterior weight-bearing knee radiographs, obtained with the SynaFlexer positioning frame (Synarc, San Francisco, California, USA), initial assessment of radiographs was undertaken by one of two trained staff at Nottingham, to dichotomise participants as osteoarthritis or non-osteoarthritis for stratification; subsequently one observer graded radiographs for knee osteoarthritis (blind to initial assessment) using a line-drawing atlas; ${ }^{28}$ classification of participants using the American College of Rheumatology (ACR) osteoarthritis classification. ${ }^{10}$

The following assessments were also undertaken at baseline and repeated at day 10, week 7 and week 13: visual analogue scale pain intensity score for the index knee; the Western Ontario McMaster Universities osteoarthritis index (WOMAC); acceptability of pain in the past $48 \mathrm{~h}$ determined by asking 'Thinking only of the pain you felt in your knee during the last $48 \mathrm{~h}$, if you were to remain with that pain for the rest of your life would that be acceptable to you?'; health-related quality of life short form 36 questionnaire; sit-to-stand test. ${ }^{29}$ In addition, reporting of adverse events (AE) and the patient global assessment (PGA) of treatment were recorded on day 10, week 7 and week 13 .

\section{Primary endpoints}

The primary short-term efficacy endpoint was the day 10 difference between groups in the WOMAC pain subscale (normalised to $0-100 \mathrm{~mm}$ scale). The primary long-term efficacy endpoint was the PGA of study medication after 13 weeks; determined by asking 'Taking into account both how your medicine worked for you and any side effects you think it caused you, how would you rate your medication as a treatment for your painful knee?' (five-point ordinal scale: 1 , excellent; 2 , good; 3, fair; 4, poor; 5, unacceptable).

The primary safety endpoint was the incidence of moderate and severe AE reported during the study period, irrespective of the relationship to the assigned treatment. All AE were assessed by the investigator, coded using the MedDRA coding dictionary (version 11.0), and were reported by severity (mild, moderate or severe) and relationship to study medication (definite, probable, possible, unlikely or none).

\section{Statistical analyses}

All statistical tests were two-tailed with significance determined by reference to the 5\% level, comparisons reported with $95 \%$ $\mathrm{CI}$ and equality of treatments was the null hypothesis. Testing for the short-term efficacy endpoint (WOMAC pain subscale) followed a closed-test procedure. First, an analysis of covariance (ANCOVA) model was fitted, with factors for treatment group, site, presence of osteoarthritis and a covariate for baseline WOMAC pain score (continuous). If the treatment group factor was significant at the $5 \%$ level, the statistical plan allowed the efficacy of two combination tablets to be compared with paracetamol. If this was significant at the $5 \%$ level, the efficacy of one combination tablet was compared with paracetamol and simultaneously two combination tablets with ibuprofen, at the $5 \%$ level; managed using Hochberg's methodology, whereby the two-sided $5 \%$ level was applied if both comparisons were significant at the $5 \%$ level, or the $2.5 \%$ level was applied if one comparison was significant. If all these comparisons were significant at the $5 \%$ level, the non-inferiority of one combination tablet was compared with ibuprofen. The primary long-term efficacy endpoint (PGA) was analysed using this same ANCOVA model. Secondary efficacy endpoints were analysed by logistic regression (binary variable) or ANCOVA (continuous variables). The incidence of AE was compared between treatment groups using the $\chi^{2}$ test.

Treatment response was defined by the OsteoArthritis Research Society International criteria; ${ }^{30}$ a reduction of at least 20 on the WOMAC pain scale, or two out of three of the following: PGA score of excellent/good; reduction of 10 or more on the WOMAC pain scale; reduction of 15 or more on the WOMAC physical function scale.

The intention-to-treat (ITT) dataset consisted of all randomly assigned participants who received one or more dose of study medication. The per-protocol (PP) dataset comprised all participants satisfying all inclusion/exclusion criteria who correctly received their allocated treatment. The safety dataset included all patients who took at least one dose of study medication. For each variable, results are given at day 10 , week 7 , week 13 and study endpoint (value for ITT dataset derived using the last observation carried forward (LOCF; last recorded post-baseline value)).

\section{Sample size calculation}

A sample size of 200 subjects/treatment group ensured that a difference of 5.5 in the WOMAC pain subscale between two 
combination tablets and paracetamol could be detected in the primary short-term efficacy outcome with a two-sample t test; assuming $90 \%$ power, a SD of 17 and a $5 \%$ significance level. ${ }^{31}$ The study was not powered to detect a difference between the combination tablet and ibuprofen.

\section{RESULTS}

\section{Participants and treatment}

In total, 892 participants from eight recruiting centres were randomly assigned to treatment (figure 1); with a mean age of 60.6 years (range 40-84 years), 51\% were men. Groups were well balanced for baseline characteristics, pain variables and quality of life scores (tables 1 and 2); minor differences were observed in the number taking concomitant medications, current smokers and gender distribution. Radiographic osteoarthritis was identified in $560(63 \%)$ and $559(63 \%)$ participants at randomisation and after formal grading, respectively; 758 (85\%) participants fulfilled ACR criteria for knee osteoarthritis.

\section{Efficacy endpoints}

Comparison of the least square means at day 10 for WOMAC pain subscale found two combination tablets offered significantly more pain relief than paracetamol ( $\mathrm{p}=0.0012$; short-term primary efficacy endpoint; ITT dataset $(n=786)$, table 1$)$. One combination tablet showed a non-significant benefit in pain relief over paracetamol and two combination tablets showed a non-significant benefit over ibuprofen. Therefore, the closed test procedure ceased. The results for the PP dataset did not differ qualitatively from those for the ITT dataset (table 1).

In the ANCOVA model, the terms for baseline score, treatment group and site were all significant $(p<0.0001, p=0.014$, $p=0.0002$, respectively); the presence of osteoarthritis was not $(p=0.38)$. At baseline, there was a gender imbalance across treatment groups; no evidence of a gender or gender-treatment effect was observed when these factors were added into the primary model (data not shown).

At study endpoint, significantly more participants taking one or two combination tablets rated their treatment as excellent or good compared with paracetamol $(\mathrm{p}=0.0152$ and $\mathrm{p}=0.0002$, respectively; long-term primary efficacy endpoint, LOCF dataset $\mathrm{n}=880$; table 2, figure 2). Treatment group was the only term statistically significant within the ANCOVA model $(p=0.002)$.

Results for secondary efficacy endpoints are summarised in table 1 (WOMAC subscale scores) and table 2 (functional, patient-based and quality of life endpoints). Compared with paracetamol, two combination tablets produced statistically significant changes in WOMAC scores for stiffness at all time points $(\mathrm{p}<0.05)$. At day 10, two combination tablets gave statistically significant improvements in physical function $(p<0.05)$ and composite score $(\mathrm{p}<0.05)$ compared with one tablet. Function (sit-to-stand test) improved significantly in participants taking two combination tablets versus paracetamol at day 10 $(p<0.05)$, week $13(p<0.05)$ and study endpoint $(p<0.01)$ and versus ibuprofen $(p<0.05)$ and one combination tablet at week 13 $(\mathrm{p}<0.05)$.

Pairwise comparisons of the short form 36 questionnaire results showed significant differences for two combination tablets over paracetamol at day 10 for bodily pain $(p=0.02)$ and general health $(p=0.04)$; at week 7 for role-physical score $(p=0.015)$ and at study endpoint for vitality $(p=0.02)$, social functioning $(p=0.03)$ and mental health $(p=0.04)$. One combination tablet was associated with significantly better scores than paraceta$\mathrm{mol}$ in physical functioning $(\mathrm{p}=0.04)$ and role-physical score $(p=0.0014)$ at week 7 and in social functioning at study endpoint $(p=0.03)$. No other significant differences were observed for combination over monotherapy.

At day 10 and study endpoint, respectively, the percentage of responders was $58.0 \%, 55.5 \%$ for two combination tablets, $51.5 \%, 57.7 \%$ for one combination tablet, $52.6 \%, 52.8 \%$ for

\begin{tabular}{|c|c|c|c|c|c|}
\hline \multirow{4}{*}{$\begin{array}{l}\text { 들 } \\
\text { हू } \\
\text { 은 }\end{array}$} & & \multicolumn{2}{|c|}{$\begin{array}{l}\text { Assessed for eligibility } \\
\qquad n=4999\end{array}$} & \multirow{4}{*}{\multicolumn{2}{|c|}{$\begin{array}{l}\text { Screen failures } n=187 \\
\text { Ineligible pain score/insufficient } \\
\text { frequency of pain (64) } \\
\text { Withdrew consent (29) } \\
\text { Abnormal laboratory values (28) } \\
\text { Required hospitalisation/prohibited } \\
\text { medication/prohibited medical } \\
\text { history/Investigator concerns (23) } \\
\text { Logistical reasons (22) } \\
\text { Ankle oedema (13) } \\
\text { Abnormal ECG (5) } \\
\text { Unknown (3) }\end{array}$}} \\
\hline & & $\begin{array}{c}\text { Screened } \\
n=1079\end{array}$ & & & \\
\hline & & & & & \\
\hline & & $\begin{array}{c}\text { Randomised } \\
n=892\end{array}$ & & & \\
\hline \multirow{3}{*}{ 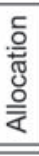 } & & & \multirow{2}{*}{\multicolumn{2}{|c|}{ Ibuprofen $n=224$}} & \\
\hline & $\begin{array}{c}2 \times \text { Combination tablet } \\
n=224\end{array}$ & $\begin{array}{c}1 \times \text { Combination tablet } \\
n=222\end{array}$ & & & Paracetamol $n=222$ \\
\hline & $\downarrow$ & $\downarrow$ & & $\downarrow$ & $\downarrow$ \\
\hline$\frac{0}{\sqrt[0]{0}}$ & $\begin{array}{l}\text { Analysed } n=204 \\
\text { Data missing (7) } \\
\text { Withdrawn (13) } \\
\text { Excluded from PP set (28) }\end{array}$ & $\begin{array}{l}\text { Analysed } n=201 \\
\text { Data missing (13) } \\
\text { Withdrawn (8) } \\
\text { Excluded from PP set (33) }\end{array}$ & $\begin{array}{l}\text { An } \\
\text { Dat } \\
\text { Wit } \\
\text { Exc }\end{array}$ & $\begin{array}{l}\text { ed } n=193 \\
\text { ssing (12) } \\
\text { wn (19) } \\
\text { d from PP set }(30)\end{array}$ & $\begin{array}{l}\text { Analysed } n=188 \\
\text { Data missing (18) } \\
\text { [Baseline missing (1)] } \\
\text { Withdrawn (16) } \\
\text { Excluded from PP set (33) }\end{array}$ \\
\hline $\begin{array}{l}\frac{m}{7} \\
\text { y } \\
\stackrel{\$}{3}\end{array}$ & $\begin{array}{l}\text { Completed } n=161 \\
\text { Withdrawn } n=63 \\
\text { Reasons*: } \\
\text {-Adverse events (41) } \\
\text { - Insufficient pain relief (18) } \\
\text { - Withdrew consent (10) } \\
\text { - Lost to follow up (4) } \\
\text {-Protocol violation (0) } \\
\text {-Death/serious AE (1) } \\
\text { - Investigator decision (0) } \\
\text { - Other (5) }\end{array}$ & $\begin{array}{l}\text { Completed } n=155 \\
\text { Withdrawn } n=67 \\
\text { Reasons*: } \\
\text {-Adverse events (31) } \\
\text { - Insufficient pain relief (21) } \\
\text { - Withdrew consent (14) } \\
\text { - Lost to follow up (2) } \\
\text { - Protocol violation (4) } \\
\text { - Death/serious AE (0) } \\
\text {-Investigator decision (2) } \\
\text { - Other (8) }\end{array}$ & $\begin{array}{l}\text { Co } \\
\text { Wi } \\
\text { Re } \\
\cdot A C \\
\cdot \text { - Ins } \\
\cdot \text { Wi } \\
\cdot \text { Lo } \\
\cdot \text { Pr } \\
\cdot \text { De } \\
\cdot \text { In } \\
\cdot \text { Ot }\end{array}$ & $\begin{array}{l}\text { eted } n=163 \\
\text { awn } n=61 \\
s^{*}: \\
\text { e events (34) } \\
\text { lent pain relief (17) } \\
\text { w consent (8) } \\
\text { follow up (4) } \\
\text { violation (0) } \\
\text { erious AE (1) } \\
\text { ator decision (3) } \\
\text { 5) }\end{array}$ & $\begin{array}{l}\text { Completed } n=136 \\
\text { Withdrawn } n=86 \\
\text { Reasons*: } \\
\text {-Adverse events (39) } \\
\text { - Insufficient pain relief (29) } \\
\text { - Withdrew consent (13) } \\
\text { - Lost to follow up (5) } \\
\text { - Protocol violation (3) } \\
\text {-Death/serious AE (2) } \\
\text { - Investigator decision (3) } \\
\text { - Other (9) }\end{array}$ \\
\hline
\end{tabular}

Figure 1 CONSORT flow diagram of participants through each stage of the trial. *Not mutually exclusive; AE, adverse event; PP, per protocol. 
Table 1 Baseline and change from baseline to day 10, week 7 and week 13 in WOMAC osteoarthritis index scale points-intention-to-treat dataset

\begin{tabular}{|c|c|c|c|c|}
\hline & Paracetamol & Ibuprofen & $1 \times$ Combination tablet & $2 \times$ Combination tablet \\
\hline \multicolumn{5}{|l|}{ WOMAC subscale points } \\
\hline \multicolumn{5}{|l|}{ Pain $^{\dagger}$} \\
\hline Baseline, mean $\pm S D(n)$ & $43.0 \pm 14.9(221)$ & $44.0 \pm 15.2(224)$ & $45.0 \pm 16.0(222)$ & $42.5 \pm 15.7(224)$ \\
\hline Day 10 & $-10.1 \pm 16.3(188)^{* *}$ & $-13.3 \pm 17.8(193)$ & $-12.8 \pm 16.7(201)$ & $-15.0 \pm 17.5(204)$ \\
\hline Week 7 & $-14.7 \pm 17.8(148)$ & $-15.0 \pm 19.7(174)$ & $-17.1 \pm 18.8(161)$ & $-18.0 \pm 20.3(173)$ \\
\hline Week 13 & $-15.9 \pm 16.3(136)$ & $-17.6 \pm 19.6(162)$ & $-16.8 \pm 19.0(151)$ & $-18.3 \pm 19.5(159)$ \\
\hline \multicolumn{5}{|c|}{ Day 10 pairwise comparisons of change in baseline for WOMAC pain subscale-primary short-term endpoint (PP dataset) } \\
\hline & Mean difference $e^{\ddagger}$ & SE & $95 \% \mathrm{Cl}$ & p Value ${ }^{\ddagger}$ \\
\hline Two combination tablets vs paracetamol & $-5.3(-5.1)$ & $1.6(1.8)$ & $-8.5,-2.1(-8.5$ to -1.6$)$ & $0.0012^{* *}\left(0.0040^{* *}\right)$ \\
\hline One combination tablet vs paracetamol & $-2.4(-2.7)$ & $1.6(1.8)$ & $-5.7,0.8(-6.2$ to 0.8$)$ & $0.1389(0.1298)$ \\
\hline Two combination tablets vs ibuprofen & $-2.2(-2.1)$ & $1.6(1.7)$ & $-5.4,1.0(-5.5$ to 1.3$)$ & $0.1787(0.2331)$ \\
\hline One combination tablet vs ibuprofen & $0.7(0.3)$ & $1.6(1.8)$ & $-2.5,3.9(-3.1$ to 3.8$)$ & $0.6772(0.8621)$ \\
\hline Day 10 & $-8.3 \pm 15.5(186)^{* * *}$ & $-10.8 \pm 14.3(186)$ & $-10.3 \pm 14.8(194)^{*}$ & $-13.1 \pm 16.5(203)$ \\
\hline Week 7 & $-11.2 \pm 16.8(145)^{*}$ & $-13.1 \pm 17.0(170)$ & $-14.1 \pm 16.2(154)$ & $-16.0 \pm 19.1(171)$ \\
\hline Week 13 & $-12.7 \pm 17.2(133)$ & $-13.0 \pm 17.1(158)$ & $-13.4 \pm 18.2(144)$ & $-14.5 \pm 18.5(156)$ \\
\hline Endpoint (LOCF) & $-9.2 \pm 17.8(211)^{*}$ & $-10.5 \pm 17.8(213)$ & $-10.9 \pm 17.4(216)$ & $-12.5 \pm 18.8(217)$ \\
\hline \multicolumn{5}{|l|}{ Stiffness $^{\dagger}$} \\
\hline Baseline, mean $\pm S D(n)$ & $51.4 \pm 21.0(221)$ & $54.4 \pm 19.8(223)$ & $54.1 \pm 22.4(221)$ & $52.4 \pm 21.7(223)$ \\
\hline \multicolumn{5}{|l|}{ Change from baseline, mean $\pm S D(n)$} \\
\hline Day 10 & $-10.7 \pm 20.5(194)^{* * *}$ & $-17.3 \pm 20.8(193)$ & $-16.4 \pm 21.1(202)$ & $-18.3 \pm 21.2(206)$ \\
\hline Week 7 & $-16.4 \pm 21.7(147)^{*}$ & $-20.8 \pm 21.9(174)$ & $-21.7 \pm 24.1(160)$ & $-23.1 \pm 23.6(173)$ \\
\hline Week 13 & $-17.0 \pm 23.3(135)^{*}$ & $-22.6 \pm 22.8(161)$ & $-22.0 \pm 25.9(152)$ & $-23.3 \pm 25.6(159)$ \\
\hline Endpoint (LOCF) & $-12.8 \pm 23.7(218)^{* *}$ & $-17.2 \pm 24.0(217)$ & $-18.2 \pm 25.1(219)$ & $-19.4 \pm 25.8(217)$ \\
\hline \multicolumn{5}{|l|}{ Composite score ${ }^{\dagger}$} \\
\hline
\end{tabular}

\footnotetext{
${ }^{*} \mathrm{p}<0.05,{ }^{* *} \mathrm{p}<0.01,{ }^{* *} \mathrm{p}<0.001$ versus two tablets of the fixed-dose tablet combination ibuprofen $200 \mathrm{mg} / \mathrm{paracetamol} 500 \mathrm{mg}$ tablet.

${ }^{\dagger}$ Normalised to $0-100 \mathrm{~mm}$ scale, lower score favourable.

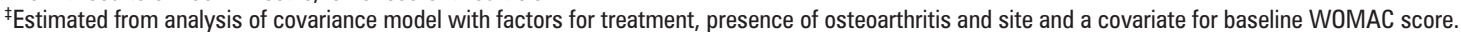

ITT, intention-to-treat; LOCF, last recorded post-baseline value; PP, per protocol; WOMAC, Western Ontario McMaster Universities osteoarthritis index.
}

ibuprofen and 45.1\%, 49.1\% for paracetamol. At day 10, there were significantly more responders taking two combination tablets versus paracetamol ( $\mathrm{p}=0.0063)$. No other statistically significant treatment differences were observed.

\section{Safety}

There were 18 serious $\mathrm{AE}, 13$ in 11 patients were treatment emergent and three were possibly related to treatment; death due to ruptured aortic aneurism, acute worsening of renal function and angina pectoris. The median incidence of all moderate and severe $\mathrm{AE}$, regardless of the relationship to treatment was 1.1 per person-days exposure in all four treatment groups. The commonest treatment-related AE were dyspepsia, diarrhoea and nausea. Compared with other treatments, a larger proportion of participants reported diarrhoea with two combination tablets; there was a higher incidence of liver function test abnormalities with paracetamol monotherapy; a higher incidence of early but transient abnormal liver function tests with paracetamol versus ibuprofen monotherapy (table 3). At study end, the incidence of drug-related AE was significantly higher in subjects taking two combination tablets compared with those taking ibuprofen monotherapy (51\% and $42 \%$, respectively; $\mathrm{p}=0.04)$. Similarly, at day $10,31 \%$ and $21 \%$ of the two combination tablet and ibuprofen groups, respectively, had a drug-related AE ( $p=0.018)$. There were no other significant differences between treatment groups in the number of drug-related AE. With respect to blood pressure, there were no significant changes from baseline to end of study in mean systolic or diastolic pressures. The values for mean change in systolic pressure $( \pm S D)$ were: $-1.0 \pm 16.5$, $1.5 \pm 13.9,-0.1 \pm 15.3$ and $1.4 \pm 15.7 \mathrm{~mm} \mathrm{Hg}$ for the paracetamol, ibuprofen, single combination tablet and two combination tablet groups, respectively; with corresponding values for diastolic pressure of: $-2.5 \pm 9.7,-0.2 \pm 9.4,-1.2 \pm 9.4$ and $-0.3 \pm 9.6 \mathrm{~mm} \mathrm{Hg}$. During the study an increase in blood pressure was reported as an $\mathrm{AE}$ in one participant in each of the ibuprofen, paracetamol and one combination tablet groups; no subjects receiving two combination tablets reported a change.

Mean haemoglobin levels decreased in all groups throughout the study (table 3). A decrease in haemoglobin level by $1 \mathrm{~g} / \mathrm{dl}$ or greater was observed in participants in all groups at day 10 ; $7.3 \%$ of those on paracetamol versus $11.3 \%$ for ibuprofen and $10.8 \%$ and $17.5 \%$ for one and two combination tablets, respectively; significantly more subjects receiving two combination tablets experienced this decrease compared with those taking 
Table 2 Results of functional, patient based and quality of life efficacy endpoints - intention-to-treat dataset

\begin{tabular}{|c|c|c|c|c|}
\hline & Paracetamol & Ibuprofen & $1 \times$ Combination tablet & $2 \times$ Combination tablet \\
\hline \multicolumn{5}{|l|}{ Sit-to-stand test, seconds, mean \pm SD (n) } \\
\hline Baseline & $21.4 \pm 33.8(209)$ & $20.5 \pm 33.2(218)$ & $19.4 \pm 11.2(210)$ & $21.8 \pm 61.5(215)$ \\
\hline Day 10 & $16.7 \pm 9.5(176)^{*}$ & $16.3 \pm 6.6(183)$ & $16.5 \pm 6.8(191)$ & $14.9 \pm 5.6(203)$ \\
\hline Week 7 & $15.6 \pm 8.5(139)$ & $15.3 \pm 5.9(168)$ & $15.3 \pm 6.0(152)$ & $14.1 \pm 6.2(171)$ \\
\hline Week 13 & $15.3 \pm 7.7(124)^{*}$ & $15.4 \pm 5.9(158)^{*}$ & $15.2 \pm 5.9(142)^{*}$ & $13.7 \pm 5.1(154)$ \\
\hline Endpoint (LOCF) & $16.7 \pm 9.4(199)^{* *}$ & $15.7 \pm 6.5(208)$ & $16.0 \pm 7.1(204)$ & $14.5 \pm 6.0(207)$ \\
\hline \multicolumn{5}{|c|}{ Acceptability of knee pain in last $48 \mathrm{~h}$, number reporting yes to acceptability question (n) } \\
\hline Baseline & $80,36.0 \%(222)$ & $83,37.1 \%(224)$ & $71,32.0 \%(222)$ & $85,37.9 \%(224)$ \\
\hline Day 10 & $119,61.0 \%(195)^{* *}$ & $125,64.1 \%(195)$ & $115,56.7 \%(203)^{* *}$ & $151,73.7 \%(205)$ \\
\hline Week 7 & $108,72.5 \%(149)$ & $118,67.8 \%(174)$ & $109,69.0 \%(158)$ & $133,75.1 \%(177)$ \\
\hline Week 13 & $95,70.9 \%(134)$ & $113,70.6 \%(160)$ & $116,76.3 \%(152)$ & $119,74.4 \%(160)$ \\
\hline Endpoint (LOCF) & $138,63.0 \%(219)$ & $139,64.1 \%(217)$ & $141,64.1 \%(220)$ & $144,65.5 \%(220)$ \\
\hline \multicolumn{5}{|c|}{ Patient global assessment ${ }^{\dagger}$, patients rating treatment as excellent or good, $n / N(\%)$} \\
\hline Day 10 & $74 / 194(38.1)^{* * *}$ & 106/194 (54.6) & $110 / 204(53.9)$ & $127 / 207(61.4)$ \\
\hline Week 7 & $81 / 149(54.4)^{*}$ & $100 / 176(56.8)$ & $96 / 161(59.6)$ & $119 / 178(66.9)$ \\
\hline Week 13 & $74 / 136(54.4)^{* *}$ & $93 / 161$ (57.8) & $93 / 153(60.8)$ & $107 / 160(66.9)$ \\
\hline At endpoint (LOCF) & $100 / 220(45.5)^{* * *}$ & $111 / 219(50.7)$ & $119 / 220(54.1)$ & $133 / 221(60.2)$ \\
\hline Endpoint (LOCF) least squares means ${ }^{\ddagger}$ & 2.97 & 2.68 & 2.69 & 2.54 \\
\hline \multicolumn{5}{|c|}{ Pairwise comparisons of patient global assessment at study endpoint_primary long-term endpoint } \\
\hline & Mean difference ${ }^{\ddagger}$ & SE & $95 \% \mathrm{Cl}$ & p Value V $^{\ddagger}$ \\
\hline Two combination tablets vs paracetamol & -0.43 & 0.12 & -0.66 to -0.20 & 0.0002 \\
\hline One combination tablet vs paracetamol & -0.28 & 0.12 & -0.51 to -0.05 & 0.0152 \\
\hline Two combination tablets vs ibuprofen & -0.14 & 0.12 & -0.37 to 0.09 & 0.2243 \\
\hline One combination tablet vs ibuprofen & 0.01 & 0.12 & -0.22 to 0.24 & 0.9539 \\
\hline
\end{tabular}

${ }^{*} \mathrm{p}<0.05,{ }^{* *} \mathrm{p}<0.01,{ }^{* * *} \mathrm{p}<0.001$ versus two tablets of the fixed-dose tablet combination ibuprofen/paracetamol.

${ }^{\dagger}$ Measured on a five-point scale where 1 is excellent, 2 is good, 3 is fair, 4 is poor, 5 is unacceptable.

${ }^{\ddagger}$ Estimated from analysis of covariance model with factors for treatment, presence of osteoarthritis and site and a covariate for baseline WOMAC score.

LOCF, last recorded post-baseline value; WOMAC, Western Ontario McMaster Universities Osteoarthritis Index.

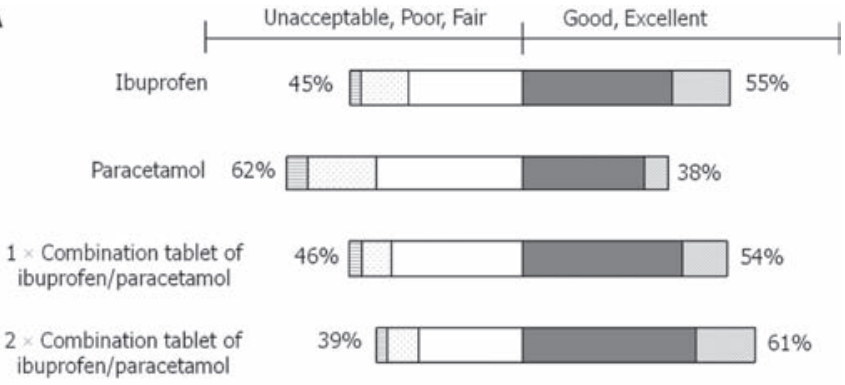

B
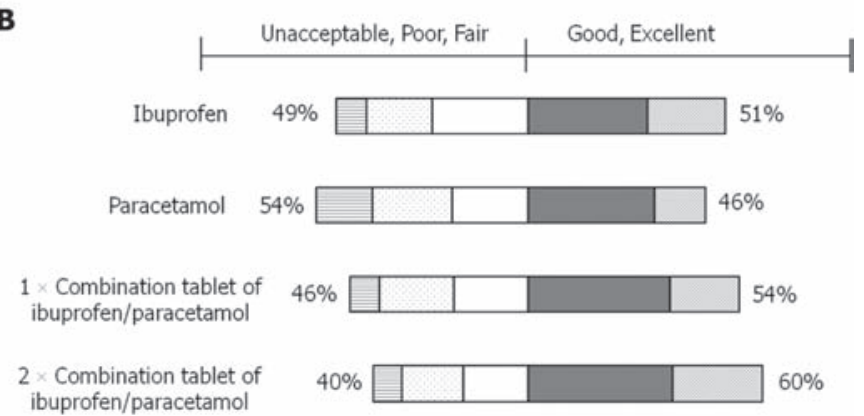

$\square$ Unacceptable $\square$ Poor $\square$ Fair $\square$ Good $\square$ Excellent

Figure 2 Bar chart of subject global assessment of treatment $(A)$ at day 10 and (B) at study endpoint (last observation carried forward; last recorded post-baseline value) —intent-to-treat dataset.

paracetamol $(p<0.002)$, but other pairwise comparisons were not significant. At 13 weeks, the proportion of subjects experiencing a $1 \mathrm{~g} / \mathrm{dl}$ or greater decrease in haemoglobin had increased further and was significantly higher in the two combination tablet group compared with the other three treatment groups ( $p<0.001) ; 20.3 \%$ on paracetamol, $19.6 \%$ on ibuprofen and $24.1 \%$ and $38.4 \%$ on one and two combination tablets, respectively (table 3 ). Other pairwise comparisons were not significant. At study end, the proportion of subjects experiencing a decrease of $2 \mathrm{~g} / \mathrm{dl}$ or greater was also significantly higher in the two combination tablet group (6.9\%), compared with those in the paracetamol $(0.9 \% ; \mathrm{p}=0.011)$, ibuprofen $(0.9 \% ; \mathrm{p}=0.001)$ and one combination tablet $(1.8 \% ; \mathrm{p}=0.0096)$ groups (table 3$)$. Of those aged 65 years and older $32.4 \%$ taking one combination tablet had a $1 \mathrm{~g} / \mathrm{dl}$ or greater decrease in haemoglobin from baseline to endpoint versus $19.9 \%$ of those aged less than 65 years; corresponding values for two combination tablets were $42.9 \%$ versus $36.0 \%$. However, age, together with baseline aspirin use and smoking were not statistically significant in a logistic regression model of subjects with a haemoglobin decrease of $1 \mathrm{~g} / \mathrm{dl}$ or greater between baseline and end of study, while the terms for treatment $(p<0.0001)$, site $(p=0.003)$ and baseline haemoglobin $(p<0.0001)$ were statistically significant. Decreases in haemoglobin were accompanied by an increase in mean platelet counts and a reduction in mean red cell volume (table 3 ), suggesting that these haemoglobin decreases may relate to blood loss.

There were seven cases of clinically significant decreases in haemoglobin: three with two combination tablets, two with one combination tablet and one each with ibuprofen and paracetamol monotherapy.

\section{DISCUSSION}

Most study participants fulfilled ACR criteria for knee osteoarthritis, ${ }^{10}$ illustrating that this is a common and underdiagnosed condition within the community. The use of two combination tablets of ibuprofen/paracetamol was associated with significant improvements in pain relief, function and patient quality of life compared with paracetamol monotherapy 
Table 3 Summary of safety evaluations: adverse events and major biochemical and haematological changes—safety dataset

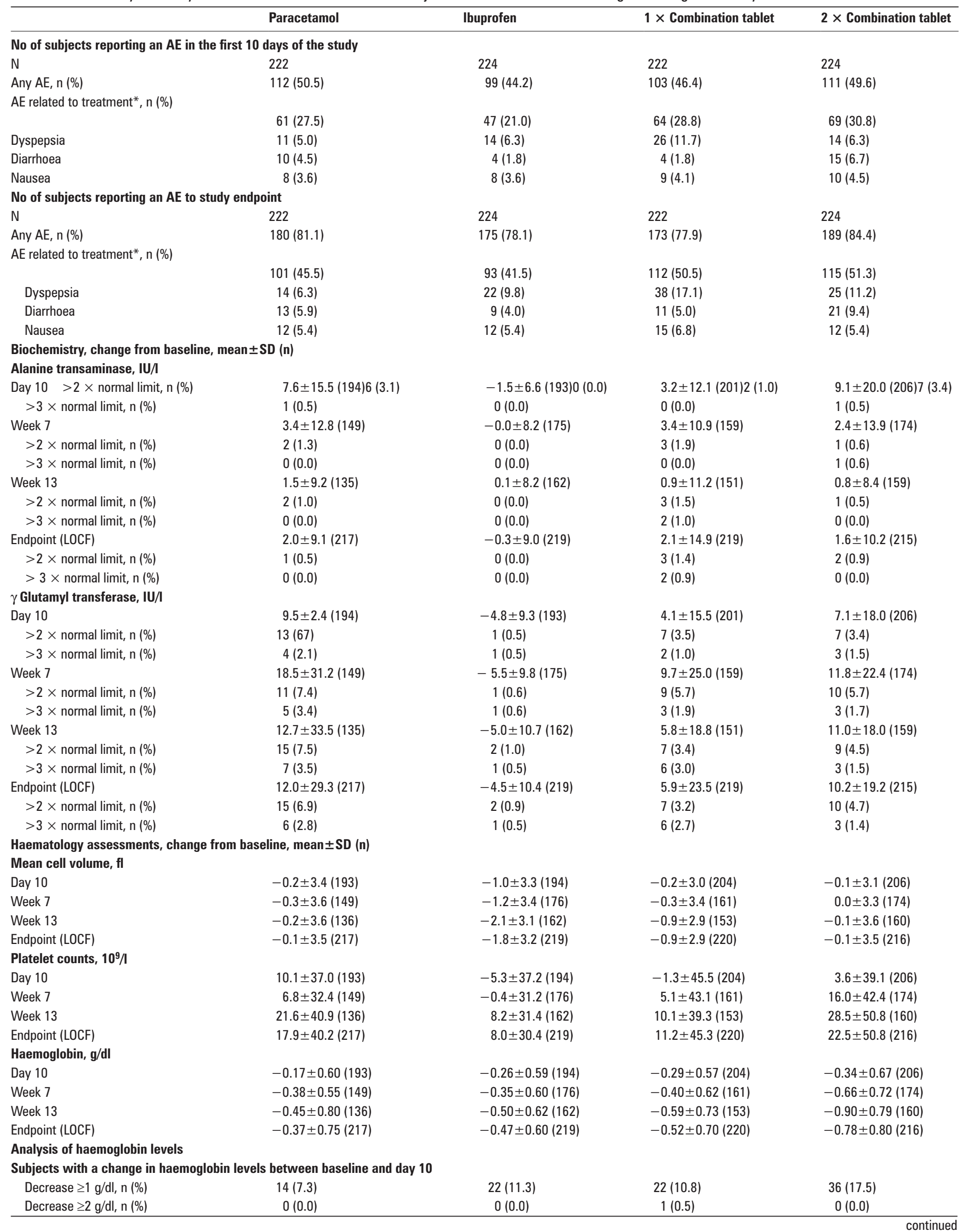


Table 3 Continued

\begin{tabular}{|c|c|c|c|c|}
\hline & Paracetamol & Ibuprofen & $1 \times$ Combination tablet & $2 \times$ Combination tablet \\
\hline \multicolumn{5}{|c|}{ Subjects with a change in haemoglobin levels between baseline and study endpoint (LOCF) } \\
\hline Decrease $\geq 1 \mathrm{~g} / \mathrm{dl}, \mathrm{n}(\%)$ & $44(20.3)$ & $43(19.6)$ & $53(24.1)$ & $83(38.4)$ \\
\hline Decrease $\geq 2 \mathrm{~g} / \mathrm{dl}, \mathrm{n}(\%)$ & $2(0.9)$ & $2(0.9)$ & $4(1.8)$ & $15(6.9)$ \\
\hline Decrease $\geq 1 \mathrm{~g} / \mathrm{dl}, \mathrm{n}(\%)$ & $37(17.1)$ & $49(22.4)$ & $49(22.3)$ & $82(38.0)$ \\
\hline Decrease $\geq 2 \mathrm{~g} / \mathrm{dl}, \mathrm{n}(\%)$ & $15(6.9)$ & $23(10.5)$ & $22(10.0)$ & $38(17.6)$ \\
\hline
\end{tabular}

* Investigator classed $\mathrm{AE}$ as definitely, probably or possibly related to treatment

$A E$, adverse event; $L O C F$, last recorded post-baseline value.

for both short and long-term use. A significantly greater proportion of patients taking two combination tablets were responders at day 10 compared with paracetamol monotherapy, but not at study end. The level of absolute pain reduction achieved with one or two combination tablets was modest but of an order considered clinically relevant. ${ }^{32} 33$ The dose of ibuprofen used in this study was moderate, reflecting the non-prescription dose, the observed improved efficacy with the combination could therefore be due to paracetamol adding further cyclooxygenase inhibition to that provided by ibuprofen alone.

This combination tablet is designed for non-prescription use, with a recommended treatment period of 3 days or less. However, many people require long-term pain relief; therefore this trial was continued to 13 weeks to assess tolerability and efficacy in the setting of arthritis management. The recruitment strategy resulted in a study population different from typical analgesic trials; participants were older, the usual use of tobacco and alcohol were allowed, many had co-morbidities and were taking concomitant medications, including aspirin.

A decrease in haemoglobin levels was observed in many participants in all treatment groups, especially in the elderly. Taken with the increase in platelet counts and reduction in mean red volumes in all groups, this reduction in haemoglobin is likely to be from occult gastrointestinal bleeding rather than from another mechanism, such as haemodilution. The relatively high incidence of diarrhoea could point to small bowel irritation as one of the sources of the bleeding. NSAIDs are well known for gastrointestinal AE but this result was unexpected for participants receiving paracetamol, in which generally no gastrointestinal risk is thought to exist. The number of participants with a decrease of $1 \mathrm{~g} / \mathrm{dl}$ or more in haemoglobin was significantly greater in those taking two combination tablets than with either monotherapy, implying a synergistic effect. Indeed, the number of participants taking two combination tablets who showed decreases in haemoglobin of $2 \mathrm{~g} / \mathrm{dl}$ or greater at study end were greater than the addition of those in the two monotherapy groups, suggesting an interaction rather than just an additive effect. The reported AE profile, however, was similar in all groups and did not differ between those with and without a haemoglobin decrease. The clinical relevance of these decreases thus remains uncertain. Nevertheless, these investigational results challenge the belief that paracetamol is the treatment of choice based on an absent/lower risk of gastrointestinal complications compared with ibuprofen. In addition, an early, but transient, rise in alanine transaminase was observed in those taking paracetamol, although this did not associate with clinical events. Nevertheless, elevation of more than twice the upper normal range in one in 50 participants taking paracetamol (compared with none of those taking ibuprofen alone) may be a cause for concern, especially as the dose was 3 g per day or less.

Previous reports on upper gastrointestinal complications associated with paracetamol are inconsistent. The risk reportedly increases with high doses ( $\geq 2$ g/day), ${ }^{34}$ when paracetamol may cause side-effects typically associated with NSAIDs; ${ }^{35}$ certainly, paracetamol does cause cyclooxygenase 2 inhibition and may act as a weak NSAID. ${ }^{19} 2035$ A retrospective cohort study of Canadian patients (>66 years), hospitalised for NSAID or paracetamol gastrointestinal-related complications, found that high-dose paracetamol carried a greater risk than low dose, and this risk increased when taken with an NSAID. ${ }^{36}$ Although our data support these findings, other studies have not confirmed paracetamol to be a risk factor for serious gastrointestinal events. ${ }^{37}$ It would seem, however, that further study of the gastrointestinal safety of paracetamol and the combination of paracetamol and NSAID is justified, especially given the widespread use of these drugs and the ability to self-medicate at the doses used in this study.

Osteoarthritis treatment guidelines recommend additive analgesia for enhanced pain relief ${ }^{10-13}$ because this may confer increased efficacy at lower doses and thus fewer side-effects. ${ }^{16}$ The long-term safety results of this study, however, do not support this rationale in adults with knee pain, although this is the first study showing such an effect and these observations require confirmation. The addition of a proton pump inhibitor is recommended to reduce gastrointestinal bleeding from NSAIDs, ${ }^{11} 12$ but this may be ineffective if the small bowel is the source. An alternative strategy is to substitute a selective cyclooxygenase 2 inhibitor for an NSAID. ${ }^{38}$ Our data raise the possibility that paracetamol has disadvantages compared with cyclooxygenase 2 inhibitors in this respect and highlight the need to define the site and mechanism of blood loss with paracetamol as a prelude to the possible use of protective strategies.

The absence of a placebo arm in this study is a limitation, but withholding analgesia for a chronic painful condition raises ethical concerns. Other caveats include: the study was not sufficiently powered to show benefit of the combination tablet over ibuprofen monotherapy; and the WOMAC scale, designed for osteoarthritis rather than knee pain, was used to determine efficacy. However, most participants fulfilled the criteria for osteoarthritis, and pain, stiffness and restricted activity are important patient-centred domains irrespective of confirmed osteoarthritis.

In conclusion, in this study ibuprofen/paracetamol combination analgesia, at non-prescription doses gave some modest improvement in pain relief for knee pain/osteoarthritis, but at the expense of an increase in side effects, namely presumed gastrointestinal bleeding. An important novel finding was that, in this population, paracetamol $3 \mathrm{~g} /$ day may cause similar degrees of blood loss (predominantly asymptomatic) as ibuprofen $1200 \mathrm{mg} /$ day, and that the combination of the two appears to be additive, or even synergistic in terms of the number of individuals with a greater than $2 \mathrm{~g} / \mathrm{dl}$ decrease in haemoglobin. These results need to be confirmed, along with their clinical relevance and identification of the site of gastrointestinal bleeding. If confirmed, this observation should lead to the re-consideration of current recommendations for oral analgesic use in osteoarthritis, 
and in chronic pain in general, and to the consideration of strategies to reduce this side-effect.

Acknowledgements The authors are grateful to Reckitt Benckiser Healthcare International Ltd for financial support; to the seven principal investigators and research nurses involved at the Synexus recruiting centres and the research metrologists in Academic Rheumatology, Nottingham, to Sally Doherty for radiographic assessments and to Professor Gary Koch, University of North Carolina at Chapel Hill, for additional statistical advice and assistance with sample size calculations.

Funding This trial was sponsored by Reckitt Benckiser Healthcare International Ltd; study no: ISRCTN77199439. Editorial assistance was provided by Debra Scates, Elements Communications Ltd, supported by Reckitt Benckiser Healthcare International Ltd.

Competing interests MD received no personal financial reimbursement for the study, but has received honoraria for attending two advisory boards for Reckitt Benckiser. CH has received honoraria for attending advisory boards for Reckitt Benckiser. SA is currently an employee of Reckitt Benckiser and IG and SR were previously employed by Reckitt Benckiser and received salary and travel expenses. $\mathrm{MG}$ and $\mathrm{NH}$ have no conflict of interest relating to this publication.

Patient consent Obtained.

Ethics approval This study was conducted with the approval of the Southampton and South West Hampshire Research Ethics Committee (A) of the NHS National Research Ethics Service.

Provenance and peer review Not commissioned; externally peer reviewed.

\section{REFERENCES}

1. Jinks C, Jordan $\mathrm{K}$, Ong BN, et al. A brief screening tool for knee pain in primary care (KNEST). 2. Results from a survey in the general population aged 50 and over. Rheumatology (Oxford) 2004;43:55-61.

2. McAlindon TE, Cooper C, Kirwan JR, et al. Knee pain and disability in the community. Br J Rheumatol 1992;31:189-92.

3. O'Reilly SC, Muir KR, Doherty M. Knee pain and disability in the Nottingham community: association with poor health status and psychological distress. Br J Rheumatol 1998;37:870-3.

4. Linsell L, Dawson J, Zondervan K, et al. Population survey comparing older adults with hip versus knee pain in primary care. Br J Gen Pract 2005;55:192-8.

5. Mäntyselkä $\mathbf{P}$, Kumpusalo $E$, Ahonen $\mathrm{R}$, et al. Pain as a reason to visit the doctor: a study in Finnish primary health care. Pain 2001;89:175-80

6. Peat G, McCarney R, Croft P. Knee pain and osteoarthritis in older adults: a review of community burden and current use of primary health care. Ann Rheum Dis 2001:60:91-7.

7. Thorstensson CA, Andersson ML, Jönsson $\mathrm{H}$, et al. Natural course of knee osteoarthritis in middle-aged subjects with knee pain: 12-year follow-up using clinical and radiographic criteria. Ann Rheum Dis 2009;68:1890-3.

8. Peat G, Thomas E, Handy J, et al. The Knee Clinical Assessment Study - CAS(K) A prospective study of knee pain and knee osteoarthritis in the general population. BMC Musculoskelet Disord 2004;5:4

9. Dieppe $\mathbf{P}$, Basler HD, Chard J, et al. Knee replacement surgery for osteoarthritis: effectiveness, practice variations, indications and possible determinants of utilization. Rheumatology (Oxford) 1999;38:73-83.

10. American College of Rheumatology. Recommendations for the medical management of osteoarthritis of the hip and knee: 2000 update. American College of Rheumatology Subcommittee on Osteoarthritis Guidelines. Arthritis Rheum 2000;43:1905-15.

11. Jordan KM, Arden NK, Doherty M, et al. EULAR Recommendations 2003: an evidence based approach to the management of knee osteoarthritis: report of a Task Force of the Standing Committee for International Clinical Studies Including Therapeutic Trials (ESCISIT). Ann Rheum Dis 2003;62:1145-55.

12. National Collaborating Centre for Chronic Conditions. Osteoarthritis: the care and management of osteoarthritis in adults. London: NICE, 2008. http://www.nice.org.uk/ nicemedia/pdf/CG59NICEguideline.pdf (accessed 30th May 2011).
13. Zhang W, Moskowitz RW, Nuki G, et al. OARSI recommendations for the management of hip and knee osteoarthritis. Part II: OARSI evidence-based, expert consensus guidelines. Osteoarthr Cartil 2008;16:137-62.

14. Towheed T, Maxwell L, Judd M, et al. Acetaminophen for osteoarthritis. Cochrane Database Syst Rev 2006;1:CD004257.

15. Wegman A, van der Windt $\mathrm{D}$, van Tulder $\mathrm{M}$, et al. Nonsteroidal antiinflammatory drugs or acetaminophen for osteoarthritis of the hip or knee? A systematic review of evidence and guidelines. J Rheumatol 2004;31:344-54.

16. Raffa RB. Pharmacology of oral combination analgesics: rational therapy for pain J Clin Pharm Ther 2001;26:257-64.

17. Vane JR, Botting RM. Mechanism of action of anti-inflammatory drugs. Scand J Rheumatol Supp/ 1996;102:9-21.

18. Bonnefont J, Courade JP, Alloui A, et al. [Antinociceptive mechanism of action of paracetamol]. Drugs 2003;63:1-4.

19. Hinz B, Cheremina O, Brune K. Acetaminophen (paracetamol) is a selective cyclooxygenase-2 inhibitor in man. FASEB J 2008;22:383-90.

20. Catella-Lawson F, Reilly MP, Kapoor SC, et al. Cyclooxygenase inhibitors and the antiplatelet effects of aspirin. N Engl J Med 2001;345:1809-17.

21. Arendt-Nielsen L, Nielsen JC, Bjerring P. Double-blind, placebo controlled comparison of paracetamol and paracetamol plus codeine-a quantitative evaluation by laser induced pain. Eur J Clin Pharmacol 1991;40:241-7.

22. Barkin RL. Acetaminophen, aspirin, or Ibuprofen in combination analgesic products Am J Ther 2001;8:433-42.

23. Beaver WT. Combination analgesics. Am J Med 1984;77:38-53.

24. Gertzbein SD, Tile M, McMurty RY, et al. Analysis of the analgesic efficacy of acetaminophen $1000 \mathrm{mg}$, codeine phosphate $60 \mathrm{mg}$, and the combination of acetaminophen $1000 \mathrm{mg}$ and codeine phosphate $60 \mathrm{mg}$ in the relief of postoperative pain. Pharmacotherapy 1986;6:104-7.

25. Skoglund LA, Skjelbred P, Fyllingen G. Analgesic efficacy of acetaminophen $1000 \mathrm{mg}$, acetaminophen $2000 \mathrm{mg}$, and the combination of acetaminophen $1000 \mathrm{mg}$ and codeine phosphate $60 \mathrm{mg}$ versus placebo in acute postoperative pain. Pharmacotherapy 1991;11:364-9.

26. Sindrup SH, Brøsen K. The pharmacogenetics of codeine hypoalgesia. Pharmacogenetics 1995;5:335-46.

27. Hochberg MC, Chang RW, Dwosh I, et al. The American College of Rheumatology 1991 revised criteria for the classification of global functional status in rheumatoid arthritis. Arthritis Rheum 1992;35:498-502.

28. Wilkinson CE, Carr AJ, Doherty M. Does increasing the grades of the knee osteoarthritis line drawing atlas alter its clinimetric properties? Ann Rheum Dis 2005; 64:1467-73.

29. Bohannon RW. Sit-to-stand test for measuring performance of lower extremity muscles. Percept Mot Skills 1995;80:163-6.

30. Dougados $\mathbf{M}$, Leclaire $\mathrm{P}$, van der Heijde $\mathrm{D}$, et al. Response criteria for clinical trials on osteoarthritis of the knee and hip: a report of the Osteoarthritis Research Society International Standing Committee for Clinical Trials response criteria initiative. Osteoarthr Cartil 2000;8:395-403.

31. Miceli-Richard C, Le Bars M, Schmidely N, et al. Paracetamol in osteoarthritis of the knee. Ann Rheum Dis 2004;63:923-30.

32. Todd KH, Funk KG, Funk JP, et al. Clinical significance of reported changes in pain severity. Ann Emerg Med 1996;27:485-9.

33. Kelly AM. The minimum clinically significant difference in visual analogue scale pain score does not differ with severity of pain. Emerg Med J 2001;18:205-7.

34. Brune K, Hinz B, Otterness I. Aspirin and acetaminophen: should they be available over the counter? Curr Rheumatol Rep 2009;11:36-40.

35. Rodriguez LAG, Hernámdez-Diaz S. The risk of upper gastrointestinal complications associated with nonsteroidal anti-inflammatory drugs, glucocorticoids, acetaminophen, and combinations of these agents. Arthritis Research 2001;3:98-101.

36. Rahme E, Barkun A, Nedjar $\mathrm{H}$, et al. Hospitalizations for upper and lower Gl events associated with traditional NSAIDs and acetaminophen among the elderly in Quebec, Canada. Am J Gastroenterol 2008;103:872-82.

37. de Vries F, Setakis E, van Staa TP. Concomitant use of ibuprofen and paracetamol and the risk of major clinical safety outcomes. Br J Clin Pharmacol 2010;70:429-38.

38. Lanas A. A review of the gastrointestinal safety data - a gastroenterologist's perspective. Rheumatology 2010;49:ii3-10. 